\title{
The need for overcorrection: evaluation of computer-assisted, virtually planned, fronto-orbital advancement using postoperative 3D photography
}

\author{
*Nicole Frank, MD, ${ }^{1}$ Joerg Beinemann, MD, ${ }^{2}$ Florian M. Thieringer, MD, DDS, MHBA, ${ }^{2}$ \\ Benito K. Benitez, MD, DDS, MHBA, ${ }^{2,5}$ Christoph Kunz, MD, DDS, ${ }^{2,4}$ Raphael Guzman, MD,, ${ }^{1,3,4}$ and \\ Jehuda Soleman, MD ${ }^{1,3,4}$
}

\begin{abstract}
Departments of ${ }^{1}$ Neurosurgery and ${ }^{2}$ Oral and Craniomaxillofacial Surgery, University Hospital of Basel; ${ }^{3}$ Division of Pediatric Neurosurgery, University Children's Hospital of Basel; and ${ }^{4}$ Faculty of Medicine and ${ }^{5}$ Department of Clinical Research, University of Basel, Switzerland
\end{abstract}

\begin{abstract}
OBJECTIVE The main indication for craniofacial remodeling of craniosynostosis is to correct the deformity, but potential increased intracranial pressure resulting in neurocognitive damage and neuropsychological disadvantages cannot be neglected. The relapse rate after fronto-orbital advancement (FOA) seems to be high; however, to date, objective measurement techniques do not exist. The aim of this study was to quantify the outcome of FOA using computer-assisted design (CAD) and computer-assisted manufacturing (CAM) to create individualized 3D-printed templates for correction of craniosynostosis, using postoperative 3D photographic head and face surface scans during follow-up.

METHODS The authors included all patients who underwent FOA between 2014 and 2020 with individualized, CAD/ CAM-based, 3D-printed templates and received postoperative 3D photographic face and head scans at follow-up. Since 2016, the authors have routinely planned an additional "overcorrection" of $3 \mathrm{~mm}$ to the CAD-based FOA correction of the affected side(s). The virtually planned supraorbital angle for FOA correction was compared with the postoperative supraorbital angle measured on postoperative 3D photographic head and face surface scans. The primary outcome was the delta between the planned CAD/CAM FOA correction and that achieved based on 3D photographs. Secondary outcomes included outcomes with and those without "overcorrection," time of surgery, blood loss, and morbidity.
\end{abstract}

RESULTS Short-term follow-up (mean 9 months after surgery; 14 patients) showed a delta of $12^{\circ}$ between the planned and achieved supraorbital angle. Long-term follow-up (mean 23 months; 8 patients) showed stagnant supraorbital angles without a significant increase in relapse. Postsurgical supraorbital angles after an additionally planned overcorrection (of $3 \mathrm{~mm}$ ) of the affected side showed a mean delta of $11^{\circ}$ versus $14^{\circ}$ without overcorrection. The perioperative and postoperative complication rates of the whole cohort $(n=36)$ were very low, and the mean $(S D)$ intraoperative blood loss was 128 (60) $\mathrm{ml}$ with a mean (SD) transfused red blood cell volume of $133(67) \mathrm{ml}$.

CONCLUSIONS Postoperative measurement of the applied FOA on 3D photographs is a feasible and objective method for assessment of surgical results. The delta between the FOA correction planned with CAD/CAM and the achieved correction can be analyzed on postoperative 3D photographs. In the future, calculation of the amount of "overcorrection" needed to avoid relapse of the affected side(s) after FOA may be possible with the aid of these techniques.

https://thejns.org/doi/abs/10.3171/2021.1.FOCUS201026

KEYWORDS fronto-orbital advancement; computer-assisted manufacturing; computer-assisted design; craniosynostosis; CAD/CAM-based craniofacial remodeling; pediatric neurosurgery; virtual planning

I $\mathrm{N}$ craniosynostosis, upregulation of osteoblastic activity leads to one or more prematurely ossified sutures. ${ }^{1}$ This results in skull deformities, and additionally, brain growth can be affected and intracranial pressure may increase with corresponding complications such as optic nerve damage. ${ }^{2-4}$ Untreated craniosynostosis may also result in psychological and neurodevelopmental disadvantages. ${ }^{5,6}$ The main challenge of fronto-orbital advancement (FOA) surgery is accurate reconstruction of the orbital bandeau and frontal bone in regard to any

ABBREVIATIONS CAD = computer-assisted design; $\mathrm{CAM}=$ computer-assisted manufacturing; $\mathrm{CBL}=$ calculated blood loss; $\mathrm{EBV}=$ estimated blood volume; FOA = frontoorbital advancement; $\mathrm{HCT}=$ hematocrit; $\mathrm{RBC}=$ red blood cell; $\mathrm{SA}=$ supraorbital angle.

SUBMITTED December 1, 2020. ACCEPTED January 7, 2021.

INCLUDE WHEN CITING DOI: 10.3171/2021.1.FOCUS201026.

${ }^{*}$ N.F. and J.B. contributed equally to this work. 
individual variation. ${ }^{7,8}$ So far, there are no standardized guidelines for the amount of advancement or overcorrection needed in FOA surgery, and realization often depends on the experience of the surgeon. In addition, relapse after FOA surgery seems to be high and a continuous issue within the literature. ${ }^{9-11}$ During the last decade, due to fast-developing technical approaches, new procedures for improved and more accurate individual presurgical planning were developed. Image-based 3D modeling using computer-assisted design (CAD) and computer-assisted manufacturing (CAM) of implants has been used to create templates for better preoperative planning of reconstruction. ${ }^{8,12-18}$ This not only results in more precise and individual fronto-orbital reconstruction but may also reduce surgical time and blood loss..$^{16}$ Our preoperative CAD/ CAM technique for planning FOA surgery was previously published. ${ }^{18}$ However, few studies present potential postoperative measures able to objectively assess the outcome of CAD/CAM-based FOA correction. The aim of this retrospective, single-center study was to assess, in an objective manner, the outcomes of CAD/CAM-based, individualized, 3D-printed templates for FOA correction of craniosynostosis, using postoperative 3D photography during follow-up.

\section{Methods}

We retrospectively analyzed all consecutive patients who underwent CAD/CAM-based FOA surgery between 2014 and 2020 at University Children's Hospital of Basel. Medical records were reviewed for clinical variables such as age at surgery, sex, type of craniosynostosis, associated syndrome (e.g., Apert, Pfeiffer, Crouzon), preoperative and postoperative hematocrit (HCT) values, estimated blood volume (EBV), transfusion rate of red blood cells (RBCs), calculated blood loss (CBL), time of surgery, duration of ICU stay, total hospitalization time, morbidity, and mortality. EBV was calculated using an estimated 80 $\mathrm{ml}$ of RBCs per kilogram of body weight and the documented body weight on the 1st day of hospitalization. The transfusion volume of RBCs was documented in the anesthetic protocol for each patient. CBL was obtained using the following formula: $\mathrm{CBL}=\mathrm{RBC}+\mathrm{EBV}-\left(\mathrm{HCT}_{\text {pre }}-\right.$ $\left.\mathrm{HCT}_{\text {post }}\right) \cdot{ }^{19,20}$ The mean CBL was compared with the RBC transfusion volume. Time of surgery was defined from incision to skin closure. All patients were transferred to the ICU postoperatively.

\section{CAD/CAM Planning and Surgical Technique}

The preoperative CAD/CAM planning and surgical technique at our institution has been previously published by our group. ${ }^{18}$ In short, after preoperative planning, the surgical templates were manufactured for each patient. The corresponding angles of the orbital bandeaus for ideal reconstruction were based on the values of the contralateral side without synostosis for patients with unilateral coronal synostosis, as well as the values of reference skulls for healthy children of the same age, sex, and ethnic group for all patients with synostosis. Correction was adapted to the type of synostosis and severity of disease; for severe cases, a larger (over-)correction was applied compared with that
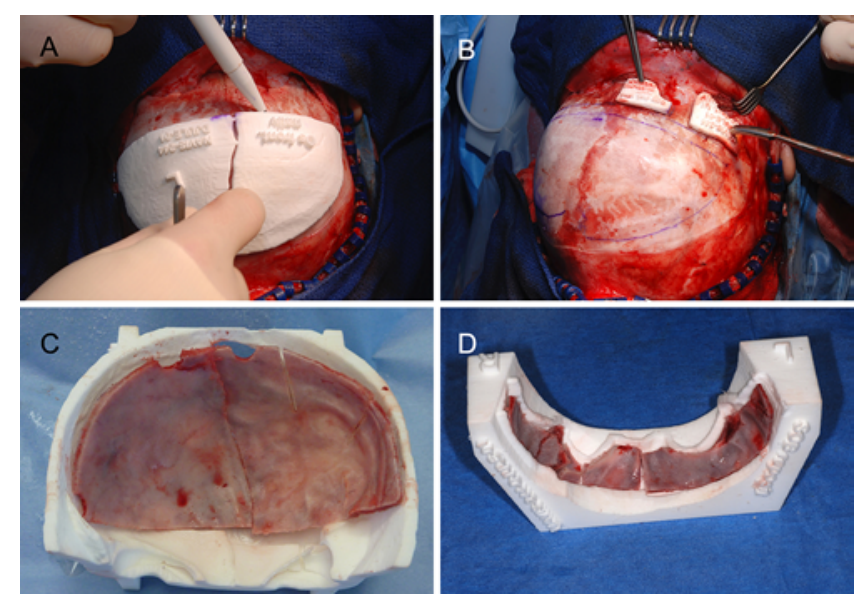

FIG. 1. Osteotomy templates of the cranial portion (A) and orbital bandeau portion (B) used to achieve accurate bone cuts based on CAD/ CAM planning. Ex vivo shaping and positioning guides for bone segments ( $C$ and $\mathbf{D})$. The guide is bowl-shaped, with ridges marking the new correct positions for different bone segments. Reproduced from Soleman et al. Neurosurg Focus. 2015;38(5):E5. ${ }^{18}$ Published with permission.

applied to less severe cases. Similarly, depending on the type of synostosis, (over-)correction was adapted (e.g., more correction for unilateral coronal synostosis than metopic synostosis).

After positioning and draping the patient, a zigzagpatterned coronal skin incision was performed to protect the pericranium and dissect it from the galea flap. The 3D-printed and sterilized individual temporal and orbital cutting guides were then placed, and the osteotomy lines were marked on the frontal and temporal bones (Fig. 1A and B). Afterward, bifrontal craniotomy was performed with minimal mobilization of the temporal muscle bilaterally, while the osteotomies of the orbital bandeau were accomplished by using a piezoelectric device (Piezosurgery system, Mectron Medical Technology). Additional osteotomies were performed separately based on the virtually planned osteotomy templates. Finally, the negative templates functioned as guides for the later fixation of the bone fragments, according to the preoperatively planned position (Fig. 1C and D). The remodeled orbital bandeau was first placed symmetrically and fixated using the SonicWeld Rx System (KLS Martin Group). Since 2016, we have intraoperatively performed, in a systematic manner, overcorrection (of $3 \mathrm{~mm}$ ) of the affected side(s) in each patient. For metopic and bilateral coronal craniosynostosis, this was applied on both sides at the edge of the resection of the orbital bandeau. Finally, the frontal bone was placed and fixated with polydioxanone sutures, a subgaleal drain was inserted, and the skin was closed in an orderly fashion.

\section{Postoperative Measurements and Evaluation Based on 3D Photographs}

All patients were scheduled for follow-up control visits, including 3D surface photographic face and head scans at different postoperative periods (approximately 3, 6, and 12 months, and then yearly), to capture the 3D representation 

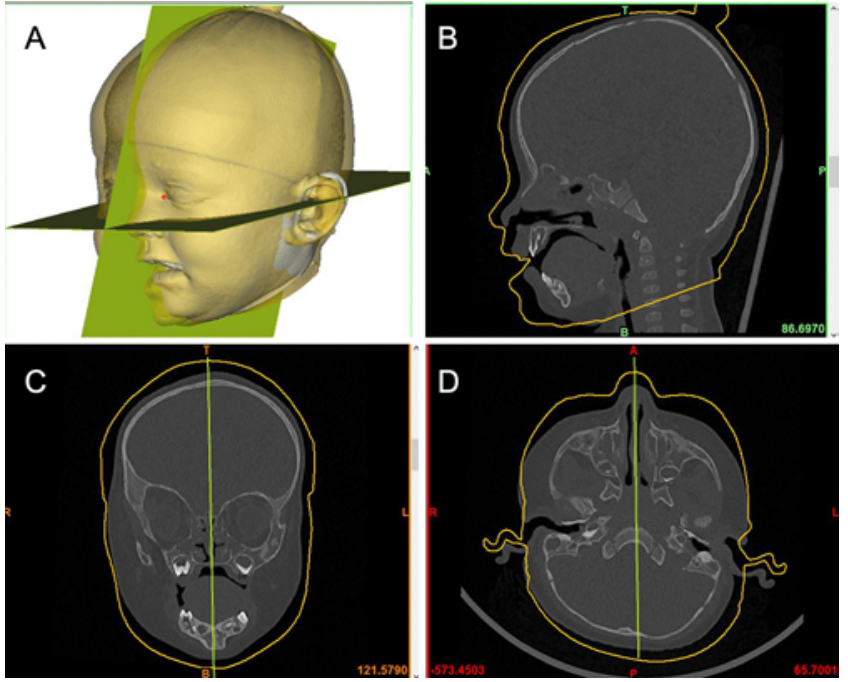

FIG. 2. Three-dimensional head and face photograph exported to the preoperative planning software for assessment and comparison with the preoperative correction plan (A). Sagittal (B), coronal (C), and axial (D) images showing the fusion of postoperative 3D photographs with preoperative CT images, after removal of 3D scanning artifacts. The softtissue surfaces were manually repositioned to match the preoperative CT scans and to allow accurate measurement of the preoperative and postoperative SAs, thereby leading to the calculation of delta SA.

of the patient's face and head (VECTRA M5 system with VECTRA Analysis Module software version 5.8, Canfield Scientific Inc.). Using automatic surface reconstruction, 3D surface data were generated and exported to the preoperative planning software (Mimics Medical version 1822, Materialise N.V.) for assessment and comparison with the preoperative correction plan (Fig. 2A). After initial removal of scanning artifacts within the 3D photographs, all relevant skin surfaces were manually repositioned to match the preoperative CT scans (Fig. 2B-D). Next, in each case, the horizontal supraorbital angle (SA) was measured native in the preoperative axial plane, directly below the upper rim of both medial supraorbital bandeau segments (Fig. 3 left). To ensure correct replicability and reliable comparability, and to avoid systematic measurement errors, lateral angle fixed points (lateral margin of each medial supraorbital bandeau segment) were defined and measured at the bone surface and the skin surface within the preoperative images (Fig. 4). The angle's center point was positioned to meet the midsagittal plane and to cover the referred structural contour outlines on both sides simultaneously (Fig. 5 left). Bone surface measurements on preoperative CT were extrapolated to the skin surface because correct measurement on postoperative 3D photographs was based on the skin surface. Therefore, these fixed points (Figs. 4 and 5) were of utmost importance to guaranteeing high accuracy when comparing preoperative CT images with postoperative 3D images. When 3D planning included an additional overcorrection (as of 2016), this distance was also defined at the lateral margins of the repositioned intermediate frontal segments of the supraorbital bandeau (Fig. 5 right). By using the defined SA measurement points, we deducted the postoperatively achieved SA correction from the preoperatively planned SA to determine delta SA (Fig. 6).

\section{Statistical Analysis}

The primary outcome measurement was delta SA. In addition, relapse (measured as delta SA between followups) was assessed over time, to determine the amount of relapse after each follow-up period and to define a time point when relapse stagnated. All other variables were considered secondary outcome measurements. Furthermore, after collecting data of the entire cohort, we categorized patients into two groups. Group 1 was treated between 2014 and 2015, and group 2 was treated between 2016 and 2020. Because we implemented a standardized overcorrection of $3 \mathrm{~mm}$ in our clinic in 2016, we have been able to compare the amount of SA correction achieved in order to evaluate the effects of this overcorrection on cosmetic results in an objective matter. We did not analyze the difference between virtually planned SA and followup for different types of craniosynostosis (e.g., metopic, coronal) due to similar preoperative planning and surgical
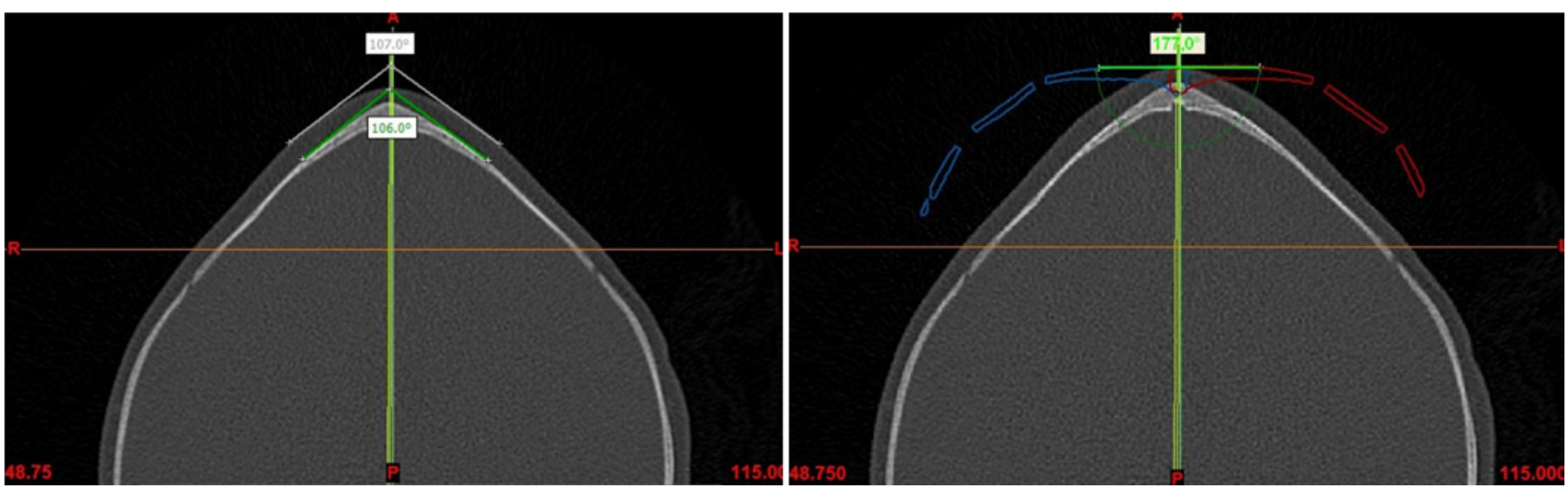

FIG. 3. Preoperative planning for metopic synostosis. Measurements of the SA in a patient with uncorrected synostosis (left), and planned preoperative correction for "ideal" SA (right). For all measurements (preoperative and postoperative), the horizontal SA was the main assessment parameter and was measured in each patient in the axial plane, directly below the upper rim of both medial supraorbital bandeau segments. 

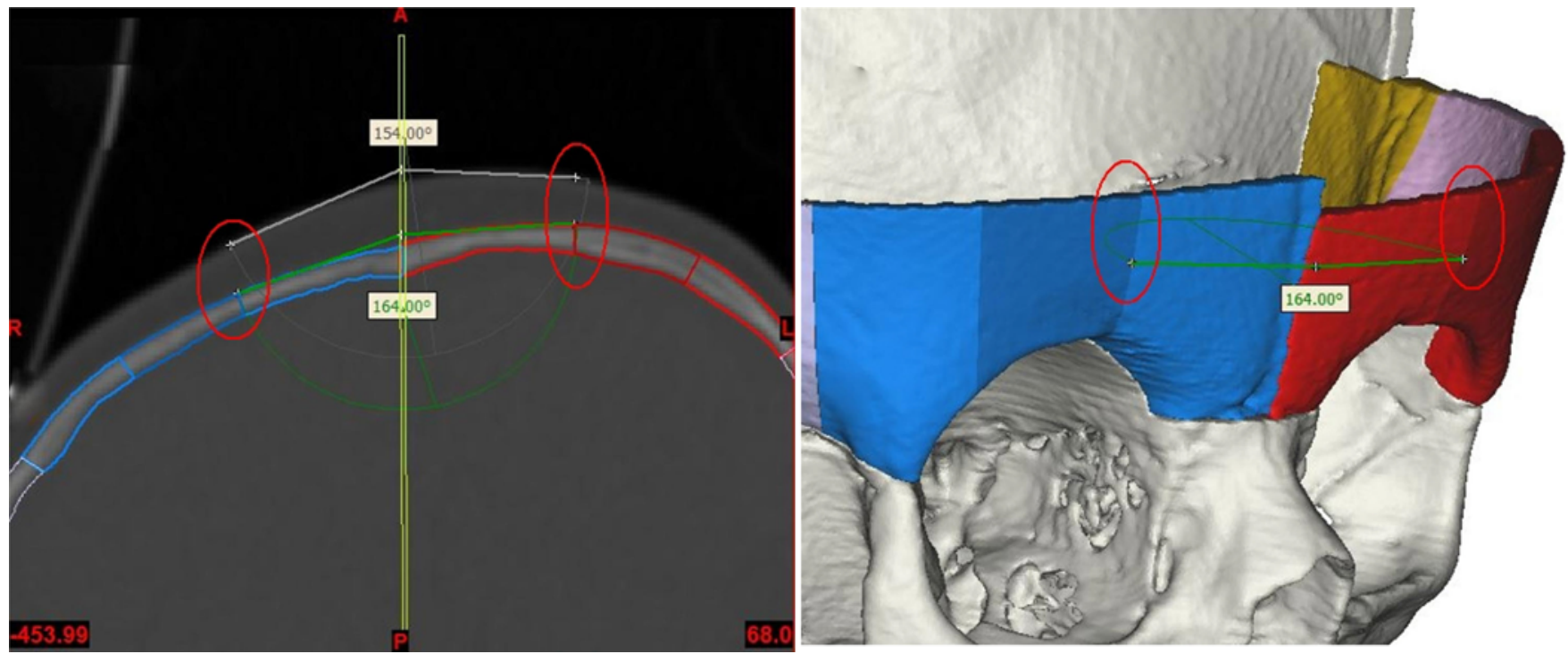

FIG. 4. Axial representation (left) and 3D reconstruction (right) of preoperative CT images, showing the defined standardized points used to measure SA. To ensure correct replicability and reliable comparability and to avoid systematic measurement errors, the lateral margin of each medial supraorbital bandeau segment (red circles) was defined as a fixed point for lateral angle measurement. These fixed points were then extrapolated to the skin surface of the CT image. Measurement errors at the skin level were minimized with systematic measurement of these fixed points, ensuring reliable comparison with skin-based postoperative 3D photographs.

procedures. In addition, the number of patients included in the cohort did not allow for such testing.

The study protocol was approved by the local ethics committee. Because of the retrospective nature of the study, the ethics committee waived the need for informed consent. All statistical analyses were performed using $\mathrm{R}$ (version 3.6.3, The $\mathrm{R}$ Foundation). All continuous variables are presented as mean with SD or median and range; nominal variables are presented as number of patients and percentage. Contingency tests were performed using the chi-square or Fisher exact test; for nonparametric tests, the Mann-Whitney U-test was used. A p value $<0.05$ was considered significant.

\section{Results}

\section{Clinical Preoperative and Intraoperative Parameters}

We included 36 patients in the analysis, with a mean (SD) age of 9 (2.7) months (range 3.6-15.8 months; median 8.3 months), including 19 males (52\%). Of these, 21
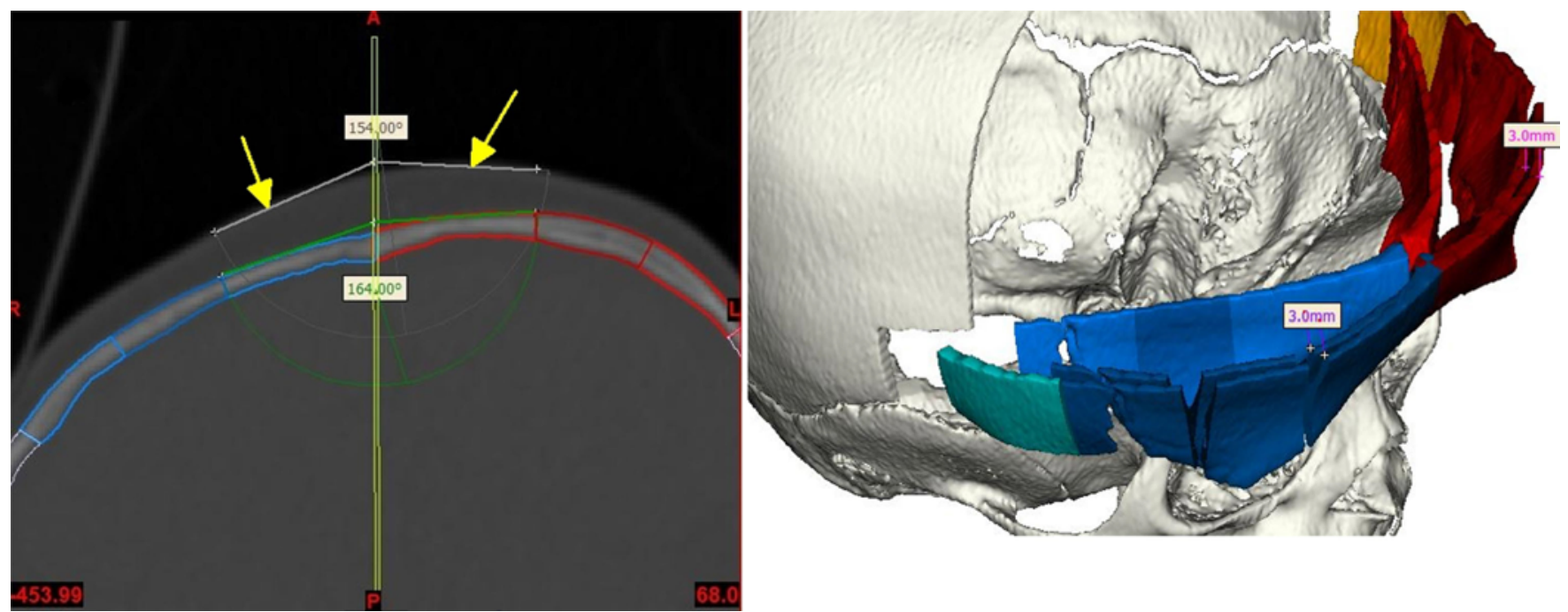

FIG. 5. Left: Image showing the importance of defining fixed points on the surfaces of the bone and skin, where the "angle wings" (arrows) should always touch the contour outlines of the measured structures. This helps avoid incorrect measurement of the SA at the level of the bone and the skin. Right: Image showing the additional overcorrection applied to all measurements since 2016, as it was applied to all preoperative calculations for visualization. 


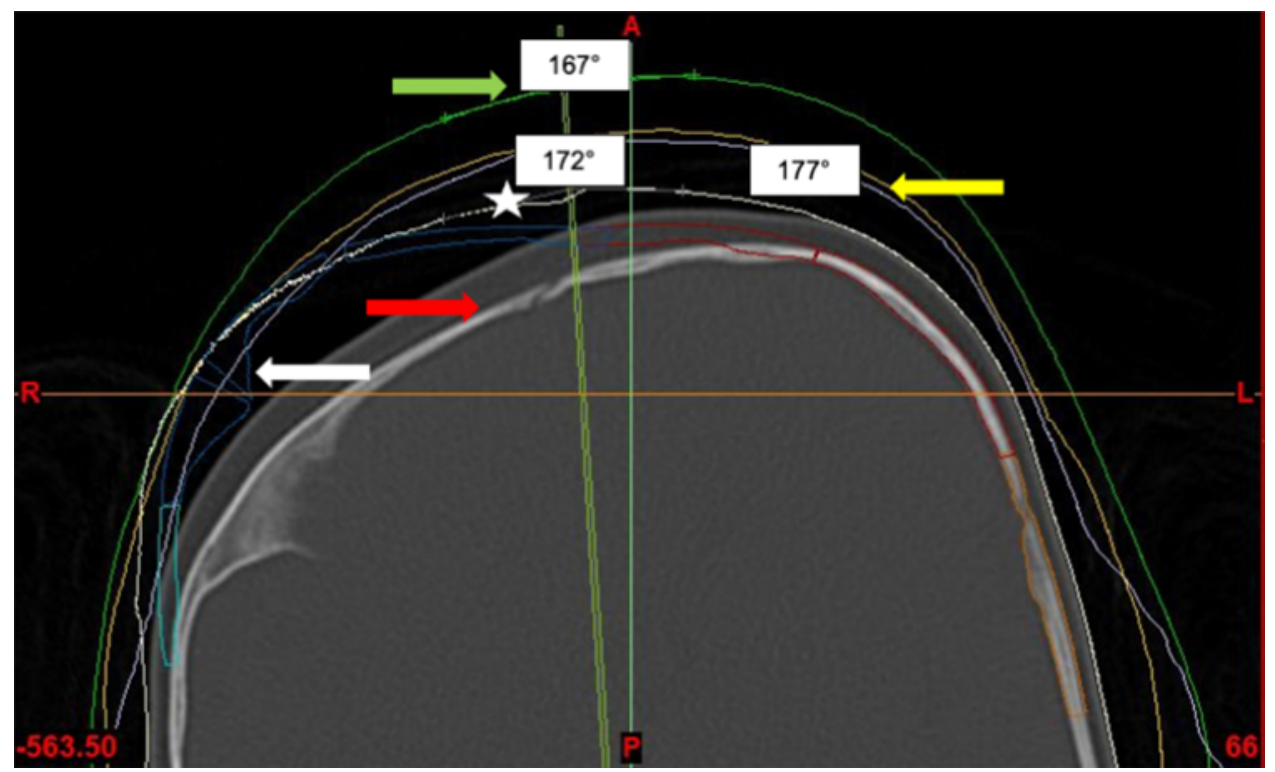

FIG. 6. Preoperative axial CT images fused with postoperative 3D photographs of patient 8 with right unilateral coronal synostosis (Table 1). The pathologically formed bone on preoperative CT (red arrow) and the planned correction (white arrow) are presented. The extrapolated correction of the skin following CAD/CAM-based correction (yellow line) with the ideal SA correction of $177^{\circ}$ (yellow arrow) is shown. The white line and white star represent correction achieved at the first follow-up, with an SA correction of $172^{\circ}$. The green line and green arrow represent correction at the second follow-up, with an SA correction of $167^{\circ}$.

patients presented with a trigonocephaly due to metopic synostosis, and 15 with plagiocephaly due to coronal synostosis. Of the 15 patients with plagiocephaly, $4(26 \%)$ had bilateral plagiocephaly and $3(20 \%)$ had syndromic synostosis. The mean (SD) operating time was 257 (45) minutes (range 180-393 minutes; median 257 minutes), the mean (SD) volume of intraoperatively transfused RBCs was 133 (67) $\mathrm{ml}$ (range 0-350 $\mathrm{ml}$; median $135 \mathrm{ml}$ ), and the mean (SD) CBL was 128 (60) $\mathrm{ml}$ (range 41-243 ml; median 120 $\mathrm{ml})$. During surgery, the mean (SD) EBV that was lost was $18 \%$ (8\%) (range 3\%-41\%; median $17 \%$ ). The mean (SD) CBL (128 [60] ml) was not significantly different from the transfused RBC volume (133 [67] ml).

The mean stay in the pediatric ICU was 1 day, and the mean hospitalization time was 6 days. One patient with syndromic craniosynostosis and known tetralogy of Fallot with impaired left and right ventricular function died on the 1st postoperative day, due to cardiac decompensation with progressive hypovolemic circulatory failure resulting in diffuse brain swelling. No other complications occurred postoperatively.

\section{Achieved FOA Correction}

Follow-up data with at least one 3D photograph were available for 14 patients (39\%) (7 in group 1 and 7 in group 2). Six patients (42\%) underwent FOA for unilateral synostosis of the coronal suture, 7 (50\%) for metopic synostosis, and $1(8 \%)$ for bilateral coronal synostosis. One patient presented with syndromic craniosynostosis (Pfeiffer syndrome). For all patients, the optimal CAD/CAM-based SA was documented for craniofacial reconstruction (Fig. 7). The mean (SD) follow-up time of the first postoperative $3 \mathrm{D}$ photograph was 9 (11) months. Table 1 shows postoper- ative SA measured on the 3D photographic head and face surface scans of all 14 patients, as well as relapse for those available. The mean (SD) delta between the planned and achieved SA of the cohort was $12.8^{\circ}\left(8^{\circ}\right)$ (range $2^{\circ}-23^{\circ}$ ). When comparing groups 1 and 2 , we saw a higher mean (SD) delta of $14^{\circ}\left(7.25^{\circ}\right)$ in group 1 (no overcorrection) compared with $11^{\circ}\left(10^{\circ}\right)$ in group $2(\mathrm{p}>0.05)$ (Table 1$)$.

At long-term follow-up (available for 8 patients; mean [SD] 23 [15] months), the mean [SD] delta was lower in group $1\left(15.8^{\circ}\left[9^{\circ}\right]\right)$ than group $2\left(19^{\circ}\left[9.54^{\circ}\right]\right)$; however, more data were available for evaluation in group 1 (Table 1). After 23 months, stagnation in the relapse of the SA between the preoperative planned and achieved corrections was seen (Table 1).

\section{Discussion}

We present, to our knowledge, the first cohort of patients with craniosynostosis who underwent repair with FOA and objective evaluation and quantification of correction based on postoperative 3D photographic head and face surface scans. Based on our data, a mean (SD) relapse or undercorrection of $12.8^{\circ}\left(8^{\circ}\right)$ occurred after FOA surgery, even when preoperative virtual planning and intraoperative implementation of CAD/CAM were done. Furthermore, it seems that standardized overcorrection of the affected side(s) may have led to less relapse and better cosmetic outcome, as assessed with 3D photographs. Our routine, which uses postoperative 3D photographic head and face surface scans, is feasible for infants and small children and spares them radiation or sedation for CT or MRI, respectively. In addition, comparison of postoperative $3 \mathrm{D}$ photographic head and face surface scans with preoperative virtually planned CAD/CAM data is feasible 

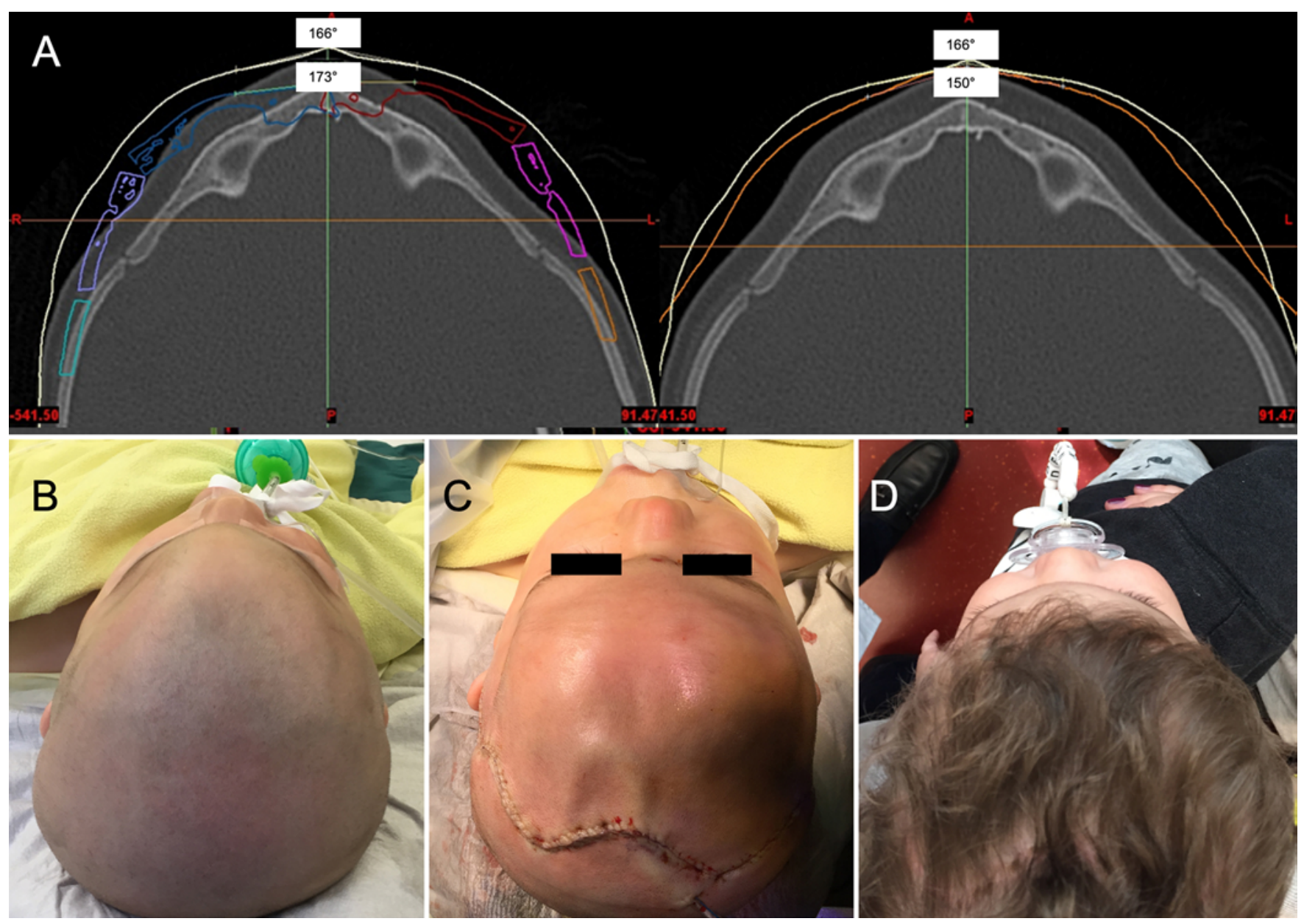

FIG. 7. A: Preoperative planning images of patient 9 with metopic synostosis (Table 1). The planning image of the bony reconstruction is shown (left), with a planned SA of $166^{\circ}$ (white line) leading to an SA of $173^{\circ}$ at the level of the bone. Axial CT image of the same patient at the first postoperative follow-up (right), showing an SA of $150^{\circ}$ at the level of the skin (orange line) based on digital reconstruction of the postoperative 3D photograph. The white line represents the planned, ideal SA based on comparison with the preoperative plan. B: Preoperative clinical photograph of the same patient. C: Postoperative bird's-eye view of the patient's head after CAD/CAM-based FOA. D: Photograph showing the clinical outcome at the first follow-up.

and provides objective measurements of the delta between the planned and achieved corrections.

Cosmetic outcome after FOA mostly depends on surgeon experience, but objective measurement tools for assessing correction after FOA are lacking. Due to continuously improving $\mathrm{CAD} / \mathrm{CAM}$ and $3 \mathrm{D}$-printing technologies, remodeling surgery for craniosynostosis is constantly improving and is an element of various studies. ${ }^{8,13-16,21,22}$ The process and administration of CAD/CAM-fabricated templates was described over 20 years ago ${ }^{23}$ and has been further developed in recent years. The orbital bandeau is the key to achieving ideal reconstruction of the frontal bone affected by congenital craniosynostosis. ${ }^{24} \mathrm{CAD} / \mathrm{CAM}$-based reconstruction helps to provide a more standardized process for consistent results. Less experienced surgeons seem to achieve better outcomes than with the "freehand" technique, and surgical revision was shown to be significantly lower in the CAD/CAM group. ${ }^{22}$ Lehner et al. compared CAD/CAM-based FOA with conventional FOA surgery and showed similar aesthetic results, although these results were based on subjective and not objective measurements
(2D photographs or postoperative CT). The CAD/CAM group, however, showed significantly shorter operating time, less blood loss, and hence lower transfusion volume. ${ }^{16}$ On the other hand, hospitalization and ICU time were reduced in the control group. ${ }^{16}$

Other studies that assessed outcome by comparing preoperative and postoperative CT scans or $2 \mathrm{D}$ photographs have been published as well. ${ }^{15,16,22}$ However, these outcomes were based on subjective and not objective measurements. Le et al. presented their objective outcome results based on 3D photographs after surgical repair of sagittal craniosynostosis, and they deemed the technique to be feasible and sufficient for outcome evaluation. ${ }^{25}$ However, studies that objectively evaluated correction achieved after FOA surgery (even when virtually planned) are lacking. In addition, relapse is a possible cosmetic burden that can occur during the follow-up period as the child grows after FOA surgery. Objectifying the amount and time of relapse after FOA surgery in the follow-up stages is an additional challenge that, to our knowledge, has not been analyzed in the literature so far. The amount of relapse seems to be influ- 
TABLE 1. Planned CAD/CAM-based parameters for FOA and follow-up parameters based on 3D photographic head and face surface scans

\begin{tabular}{|c|c|c|c|c|c|c|c|c|}
\hline \multirow[b]{2}{*}{ Variable } & \multirow{2}{*}{$\begin{array}{l}\text { Patient } \\
\text { No. }\end{array}$} & \multirow[b]{2}{*}{ CAD/CAM-Based SA } & \multicolumn{2}{|c|}{ 1st Follow-Up* } & \multicolumn{2}{|c|}{ 2nd Follow-Up† } & \multirow{2}{*}{$\begin{array}{c}\text { 3rd Follow-Up } \neq \\
\text { SA }\end{array}$} & \multirow{2}{*}{$\begin{array}{c}\text { 4th Follow-Up§ } \\
\text { SA }\end{array}$} \\
\hline & & & SA & Relapse & SA & Relapse & & \\
\hline \multirow{7}{*}{$\begin{array}{l}\text { Group } 1 \\
(n=7) \mathbb{T}\end{array}$} & 1 & 177 & 164 & 13 & 160 & 17 & 162 & 162 \\
\hline & 2 & 177 & 162 & 15 & & & & \\
\hline & 3 & 162 & 160 & 2 & 162 & 0 & 160 & 160 \\
\hline & 4 & 165 & 156 & 9 & & & & \\
\hline & 5 & 163 & 142 & 21 & 142 & 21 & 143 & 144 \\
\hline & 6 & 164 & 146 & 18 & 143 & 21 & 145 & 145 \\
\hline & 7 & 171 & 148 & 23 & 151 & 20 & & \\
\hline Mean & & & & 14 & & 15.8 & & \\
\hline Median & & & & 15 & & 20 & & \\
\hline SD & & & & 7.25 & & 9 & & \\
\hline \multirow{7}{*}{$\begin{array}{l}\text { Group } 2 \\
(n=7)^{\star *}\end{array}$} & 8 & 177 & 172 & 5 & 167 & 10 & 168 & \\
\hline & 9 & 166 & 150 & 16 & 148 & 18 & & \\
\hline & 10 & 174 & 146 & 28 & & & & \\
\hline & 11 & 177 & 157 & 20 & 148 & 29 & & \\
\hline & 12 & 167 & 171 & 4 & & & & \\
\hline & 13 & 172 & 174 & 2 & & & & \\
\hline & 14 & 175 & 171 & 4 & & & & \\
\hline Mean & & & & 11 & & 19 & & \\
\hline Median & & & & 5 & & 18 & & \\
\hline SD & & & & 10.21 & & 9.54 & & \\
\hline$p$ value & & & & 0.501 & & 0.596 & & \\
\hline
\end{tabular}

Values are shown in degrees, unless indicated otherwise.

${ }^{*}$ Mean 9 months.

† Mean 23 months.

$\ddagger$ Mean 32 months.

$\S$ Mean 46 months.

IT Treated between 2014 and 2015.

** Treated between 2016 and 2020 .

enced by surgery earlier than 6 months of age and type of synostosis. ${ }^{9,26}$ The greatest amount of relapse seems to occur during the first 12 months after surgery., ${ }^{9,27}$ Nevertheless, our reported data showed an ongoing tendency for relapse until our second follow-up at a mean of 23 months after surgery. Thereafter, according to our results, relapse did not seem to occur (Table 1). In their series of patients who underwent strong overcorrection and 5-year followup, Bennett et al. reported that only 3\% of patients had relapse that needed additional surgical intervention after approximately 6.2 years. ${ }^{11}$ In our cohort, surgery due to relapse was not indicated; however, further long-term data are necessary to reinforce our observation.

At our clinic, we used postoperative imaging with 3D photography to evaluate achieved correction, amount of relapse (or undercorrection achieved during surgery), and benefit of overcorrection in FOA surgery. An advantage of 3D photographic head and face surface scans is their feasibility and easy application to infants and small children. Furthermore, patients are spared radiation or sedation, and the images can be assessed and compared with preoperatively planned images (based on CT). In addition, 3D photography is less time consuming than other imaging modalities and can be done within the time frame of a routine postoperative clinical checkup. In 2016, we adapted our intraoperative technique to routinely add an overcorrection of $3 \mathrm{~mm}$ to the measured and planned SA. This allowed us to compare the short-term and long-term results of these two groups, based on 3D photographic head and face surface scans, in an objective manner. Although there was no significant difference between the groups, the group that received overcorrection (group 2) showed a lower tendency for relapse (of $3^{\circ}$ ) in the short-term followup period than the group without overcorrection (group 1) (Table 1). This standardized procedure provides a way for us to further analyze ideal overcorrection and compare best-possible aesthetic results in future studies. We believe that objective measurements from 3D photographic head and face surface scans and comparison with the preoperatively created virtual correction plan will help us to improve the cosmetic results of these surgeries and minimize the rate of fronto-orbital relapse.

\section{Limitations}

This retrospective study is subject to all the limitations inherent to such work. First, due to the rather small study cohort, differentiation of cosmetic outcomes according to 
craniosynostosis type and severity was not possible. Assessment of differences in cosmetic outcomes and possibly the need for overcorrection of the affected side(s) for specific types of craniosynostosis should be a subject of future studies. Second, comparison of virtual CAD/CAM-based planning based on CT with postoperative 3D photographic head and face surface scans was difficult at times and may have been slightly imprecise. This difficulty was due to the fact that comparing two different image modalities creates discrepancies (patient position, artifacts, interpolation algorithm). Third, patients were significantly older when the 3D photographs were obtained than when preoperative CT was performed, especially at the long-term follow-up. Therefore, angles may have been affected by these aspects. Application of standardized measurements with well-defined landmarks partially compensated for this. The horizontal SA served as a reliable, comparable, and recordable parameter in the analysis of diverse data modalities measured under different investigatory conditions in real patients at different times. Moreover, SA is always measurable, independent of age, growth, or available landmarks, and it is also nonsensitive with respect to artifacts. Although inaccuracies may have been registered because of incorrect positioning of the angle's fixed points, and the possible influence of the investigators exists, these inaccuracies were minimal and did not affect the final calculations. Unfortunately, other postoperative landmarkbased values are not available with surface scans. Fourth, image processing and comparison is time consuming and depends on the knowledge of the investigators. The applied software may limit extrapolation of our method and technique to other clinics, although different software solutions and packages are available and applicable. Finally, our long-term follow-up data consisted of only a small number of complete sets; therefore, these data need to be interpreted with caution, especially regarding relapse tendency. This is the first study to describe a method for objective assessment of correction, relapse rate, and overcorrection after FOA surgery. This represents a strength of this study, but future studies with larger cohorts are needed to underline and confirm our results.

\section{Conclusions}

Objective postoperative evaluation of FOA for craniosynostosis using 3D photographic head and face surface scans is feasible in infants and children. Based on our results, correction with FOA based on the CAD/CAM technique leads to good results, although there was a difference between virtually planned correction and postoperative achieved correction. This may have been due to postoperative relapse or the intraoperative difficulty of achieving the exact correction as planned. Overcorrection of the affected side(s) seems to lead to better results, but the exact amount of overcorrection needed remains to be determined. It seems that relapse rarely occurs after 2 years of followup, which may guide future follow-up regimens. With the help of objective postoperative assessment using 3D photographic head and face surface scans, we will potentially be able to assess cosmetic outcomes and calculate the ideal amount of overcorrection needed in FOA surgery.

\section{References}

1. Jin SW, Sim KB, Kim SD. Development and growth of the normal cranial vault: an embryologic review. J Korean Neurosurg Soc. 2016;59(3):192-196.

2. Renier D, Sainte-Rose C, Marchac D, Hirsch JF. Intracranial pressure in craniostenosis. J Neurosurg. 1982;57(3):370-377.

3. Wiegand C, Richards P. Measurement of intracranial pressure in children: a critical review of current methods. Dev Med Child Neurol. 2007;49(12):935-941.

4. Pattisapu JV, Gegg CA, Olavarria G, et al. Craniosynostosis: diagnosis and surgical management. Atlas Oral Maxillofac Surg Clin North Am. 2010;18(2):77-91.

5. Kunz M, Lehner M, Heger A, et al. Neurodevelopmental and esthetic results in children after surgical correction of metopic suture synostosis: a single institutional experience. Childs Nerv Syst. 2014;30(6):1075-1082.

6. Osborn AJ, Roberts RM, Mathias JL, et al. Cognitive, behavioral and psychological functioning in children with metopic synostosis: a meta-analysis examining the impact of surgical status. Child Neuropsychol. 2019;25(2):263-277.

7. Hochfeld M, Lamecker H, Thomale UW, et al. Frame-based cranial reconstruction. J Neurosurg Pediatr. 2014;13(3): 319-323.

8. Pappa H, Richardson D, Webb AAC, May P. Individualized template-guided remodeling of the fronto-orbital bandeau in craniosynostosis corrective surgery. J Craniofac Surg. 2009; 20(1):178-179.

9. Lwin CTTJW, Richardson D, Duncan C, May P. Relapse in fronto-orbital advancement: a pilot study. J Craniofac Surg. 2011;22(1):214-216.

10. Fearon JA, Ruotolo RA, Kolar JC. Single sutural craniosynostoses: surgical outcomes and long-term growth. Plast Reconstr Surg. 2009;123(2):635-642.

11. Bennett KG, Liang F, Ranganathan K, et al. Surgical hypercorrection of trigonocephaly: evaluation of surgical outcomes. J Craniofac Surg. 2018;29(1):56-61.

12. Ganesh P, Mahipathy SRRV, Vivakaran TTR, et al. Traditional versus virtual surgery planning of the fronto-orbital unit in anterior cranial vault remodeling surgery. J Craniofac Surg. 2020;32(1):285-289.

13. Elbanoby TM, Elbatawy AM, Aly GM, et al. 3D printing guided surgery in the treatment of unicoronal craniosynostosis orbital dysmorphology. Oral Maxillofac Surg. 2020;24(4): 423-429.

14. Burge J, Saber NR, Looi T, et al. Application of CAD/CAM prefabricated age-matched templates in cranio-orbital remodeling in craniosynostosis. J Craniofac Surg. 2011;22(5): 1810-1813.

15. Khechoyan DY, Saber NR, Burge J, et al. Surgical outcomes in craniosynostosis reconstruction: the use of prefabricated templates in cranial vault remodelling. J Plast Reconstr Aesthet Surg. 2014;67(1):9-16.

16. Lehner M, Wendling-Keim D, Kunz M, et al. On-site CAD templates reduce surgery time for complex craniostenosis repair in infants: a new method. Childs Nerv Syst. 2020;36(4): 793-801.

17. Rodt T, Schlesinger A, Schramm A, et al. 3D visualization and simulation of frontoorbital advancement in metopic synostosis. Childs Nerv Syst. 2007;23(11):1313-1317.

18. Soleman J, Thieringer F, Beinemann J, et al. Computerassisted virtual planning and surgical template fabrication for frontoorbital advancement. Neurosurg Focus. 2015;38(5):E5.

19. Brecher ME, Monk T, Goodnough LT. A standardized method for calculating blood loss. Transfusion. 1997;37(10): 1070-1074.

20. Schneider C, Kamaly-Asl I, Ramaswamy V, et al. Neoadjuvant chemotherapy reduces blood loss during the resection of pediatric choroid plexus carcinomas. J Neurosurg Pediatr. 2015;16(2):126-133. 
21. Lethaus B, Gruichev D, Gräfe D, et al. "Black bone": the new backbone in CAD/CAM-assisted craniosynostosis surgery? Acta Neurochir (Wien). Published online June 9, 2020. doi: 10.1007/s00701-020-04445-z

22. Cho RS, Lopez J, Musavi L, et al. Computer-assisted design and manufacturing assists less experienced surgeons in achieving equivalent outcomes in cranial vault reconstruction. J Craniofac Surg. 2019;30(7):2034-2038.

23. Vander Sloten J, Degryse K, Gobin R, et al. Interactive simulation of cranial surgery in a computer aided design environment. J Craniomaxillofac Surg. 1996;24(2):122-129.

24. Salyer KE, Hall JD. Bandeau - the focal point of frontocranial remodeling. J Craniofac Surg. 1990;1(1):18-31.

25. Le MB, Patel K, Skolnick G, et al. Assessing long-term outcomes of open and endoscopic sagittal synostosis reconstruction using three-dimensional photography. J Craniofac Surg. 2014;25(2):573-576.

26. Selber JC, Brooks C, Kurichi JE, et al. Long-term results following fronto-orbital reconstruction in nonsyndromic unicoronal synostosis. Plast Reconstr Surg. 2008;121(5):251e-260e.

27. Klausing A, Röhrig A, Lüchters G, et al. Follow-up study to investigate symmetry and stability of cranioplasty in craniosynostosis-introduction of new pathology-specific parameters and a comparison to the norm population. J Craniomaxillofac Surg. 2019;47(9):1441-1448.

\section{Disclosures}

Dr. Benitez received a grant from the Department of Surgery, University Hospital of Basel, to complete his PhD studies. The funding source was not involved in designing the study or collecting, analyzing, or interpreting data.

\section{Author Contributions}

Conception and design: Frank, Soleman. Acquisition of data: Frank, Beinemann, Thieringer, Benitez. Analysis and interpretation of data: Frank, Beinemann, Kunz, Guzman, Soleman. Drafting the article: Frank, Beinemann. Critically revising the article: all authors. Reviewed submitted version of manuscript: all authors. Approved the final version of the manuscript on behalf of all authors: Frank. Statistical analysis: Frank, Soleman. Administrative/technical/material support: Thieringer, Benitez, Kunz, Guzman. Study supervision: Soleman.

\section{Correspondence}

Nicole Frank: University Hospital of Basel, Switzerland. frank.nicole1991@gmail.com. 